\title{
La ville en live. Itinéraires numériques et artistiques à travers le patrimoine urbain
}

Live city. Digital and artistic itineraries through urban heritage sites

Emmanuelle Lambert, Jean-Thierry Julia et Julie Deramond

\section{(2) OpenEdition}

Journals

Édition électronique

URL : http://journals.openedition.org/edc/6466

DOI : $10.4000 /$ edc. 6466

ISSN : 2101-0366

Éditeur

Université Lille-3

Édition imprimée

Date de publication : 1 décembre 2015

Pagination : 113-128

ISBN : 978-2-917562-14-7

ISSN : $1270-6841$

Référence électronique

Emmanuelle Lambert, Jean-Thierry Julia et Julie Deramond, « La ville en live. Itinéraires numériques et artistiques à travers le patrimoine urbain », Études de communication [En ligne], 45 | 2015, mis en ligne le 01 décembre 2017, consulté le 20 avril 2019. URL : http://journals.openedition.org/edc/6466 ; DOI : $10.4000 /$ edc.6466 


\section{La ville en live}

Itinéraires numériques et artistiques à travers le patrimoine urbain

Live city

Digital and artistic itineraries through urban heritage sites

Emmanuelle Lambert

UPS, LERASS - équipe MICS, Université de Toulouse emmanuelle.lambert@iut-tlse3.fr

Jean-Thierry Julia

UPS, LERASS - équipe MICS, Université de Toulouse jean-thierry.julia@iut-tlse3.fr

Julie Deramond

UPS, LERASS - équipe MICS, Université de Toulouse julie.deramond@iut-tlse3.fr 
L'article interroge les modalités de médiation du patrimoine urbain, à travers trois dispositifs en ligne, relatifs à Toulouse, Lens et Marseille. Ces dispositifs construisent-ils des médiations singulières, susceptibles de modifier notre regard sur le patrimoine? Leur analyse permet de mettre au jour plusieurs types d'interaction utilisateur/ dispositif, entre relative passivité devant un audiovisuel et activité vouée à l'exploration d'un patrimoine urbain numérique. Nous étudions encore comment des formes d'art, au creux de ces projets, peuvent contribuer à exprimer différemment la ville. Nous analysons enfin les processus de valorisation et de représentation du patrimoine urbain à l'œuvre dans de tels dispositifs.

Mots-clés : médiation, patrimoine, ville, art, mutimédia.
This article considers modes of urban heritage mediation through analysis of three online devices evoking the cities of Toulouse, Lens and Marseille. What is the originality of these devices in terms of mediation? Can they change our view of cultural heritage? The three devices allow us to consider several types of interaction between user and device ranging from relatively passive reception of audiovisual messages to active exploration of digital urban heritage. We also investigate the ways in which the art forms integrated into these projects help to express the city differently. Lastly, we analyze the processes of urban heritage valorization and representation in these devices.

Keywords: mediation, heritage, city, art, multimedia. 
Le patrimoine se découvre aujourd'hui à l'aune de la réalité augmentée, de visites virtuelles ou de reconstitutions en 3D, jouant souvent sur la mise en scène des monuments. Dans ce contexte, nous souhaiterions montrer que la dimension numérique ouvre aussi à d'autres représentations que les images de synthèse, d'autres écritures, d'autres modalités symboliques d'un rapport au passé, ainsi qu'au présent. Cette réflexion s'attache à l'analyse de modalités de médiation patrimoniales, à l'œuvre dans trois dispositifs interactifs dans lesquels médiation technique et discours de médiation s'articulent autour de variations artistiques et d'interprétations créatives du patrimoine. Ces médiations interrogent aussi en creux l'implication de différents acteurs (artistes, habitants, voire internautes) dans la construction ou la transformation du regard sur le patrimoine. Dans quelles mesures ces dispositifs construisent-ils des médiations singulières et redessinent-ils les contours du patrimoine urbain?

Les trois dispositifs étudiés émanent respectivement des villes de Toulouse, Lens et Marseille. Initié en 2013, Toulouse is beautiful' se propose de faire "découvrir un lieu du patrimoine toulousain, investi par un groupe de musique en session live». Visites musicales et vidéo à la manière de clips, il s'agit ici d' "offrir un nouveau regard sur des lieux emblématiques de la ville rose»: la musique contribue à insuffler une nouvelle vie au lieu, la vidéo quant à elle permet de révéler un nouvel angle de vue. A l'occasion de l'inauguration du Louvre-Lens en 2012, le Louvre et Arte ont souhaité quant à eux « raconter un autre versant de l'aventure du nouveau musée», ce qui aboutira à la mise en ligne de Lens, vous voyez le tableau ${ }^{2}$. Ce projet inaugure une collection de Carnets de ville, qualifiée par les concepteurs de dispositifs de "prospective urbaine", enrichie depuis par une deuxième réalisation, Marseille, le mistral urbain ${ }^{3}$ : pour chacun d'eux, ces carnets consistent en une longue fresque dessinée, enrichie par les ambiances sonores de la ville. Nous investirons toutefois Marseille par un autre dispositif, Promenade nocturne à Marseille ${ }^{4}$, sous-titré Écoutez, les ruelles vont vous parler, proposé dans le cadre de Marseille 2013-Capitale européenne de la culture. La promenade plonge l'internaute dans le cours Julien, quartier des artistes et des populations parfois marginales, où le street art est roi.

La méthode d'investigation du corpus a consisté en une analyse de contenus, notamment dans sa déclinaison sémio-pragmatique. L'étude a porté sur les dispositifs accessibles sur l'internet et s'est attachée aux éléments textuels, visuels et sonores, mis à disposition au fil des consultations. Au-delà de la

1 http://toulouseisbeautiful.com. Sur les douze clips prévus à terme, nous analyserons les sept accessibles en ligne à la date du $1^{\text {er }}$ mars 2015. Leur réalisation est signée Mamy Scopitone et Betty Book Production.

2 http://louvre-lens.arte.tv/fr/

3 http://mistral-urbain.arte.tv/fr/, carnet ayant quant à lui accompagné l'ouverture du Mucem en 2013.

4 http://promenadenocturne.withgoogle.com. Le dispositif est une réalisation de Google, inspirée des Promenades sonores de Radio Grenouille à Marseille. 
comparaison de différentes visites virtuelles, l'idée est de réfléchir à la diversité des représentations et modalités de médiation du patrimoine via le numérique: nous emprunterons les parcours multimédias, pour y décrypter les dimensions de l'interaction entre utilisateur et dispositif; puis nous décomposerons les paysages visuels et sonores, pour montrer comment des formes d'art peuvent exprimer différemment la ville et participer à sa médiation; nous analyserons enfin les processus de valorisation et de représentation du patrimoine urbain.

\section{1 \\ Parcours multimédias : visiter la ville virtuellement}

Toulouse is beautiful relève pour l'essentiel de séquences vidéo réalisées dans l'enceinte de différents monuments toulousains, comme autant de clips de quelques minutes: à la suite de quelques «cartons» en guise de médiation des lieux $x^{5}$, l'image alterne vues du site et prestation musicale du groupe. Dans Lens, vous voyez le tableau, le visuel principal réside en une "fresque " - bande dessinée à la façon de la Tapisserie de Bayeux -, juxtaposition de dessins ${ }^{6}$, scènes ou portraits d'habitants. Cette fresque occupe la quasi totalité de l'écran, reléguant à sa marge quelques boutons fonctionnels. Enfin, dans Promenade nocturne à Marseille, outre la courte introduction vidéo où paraît l'initiatrice du projet ${ }^{7}$, ou encore quelques éléments paratextuels, ne s'offre au regard qu'une succession de photographies, images fixes immersives (vues $360^{\circ}$ ), restituant les ruelles marseillaises autour du cours Julien. Ainsi, le registre de l'image oppose déjà images animées toulousaines et images fixes: défilantes à Lens, et pour l'heure figée, quant aux photos marseillaises.

Une autre modalité de communication, le son, révèle à son tour un même jeu d'oppositions. Sur le site toulousain, le début de la consultation est muet, et la bande-son exclusivement musicale des vidéos revêt ensuite toute son importance, voire est au cœur de la construction filmique: sur la durée de la prestation musicale, vient en effet s'articuler l'ensemble des visuels, avec force raccords, plans de coupe, etc. (figure 1). Hors ces clips, aucune parole, et seuls quelques bruits d'ambiance viennent souligner le silence, avant et après la musique. des édifices.

6 Par Will Argunas, dessinateur et scénariste, originaire du Nord de la France.

7 Julie de Muer, artiste, productrice, collabore à Radio Grenouille, à Marseille; elle est à l'initiative des "Promenades sonores», http://www.promenades-sonores.com. 


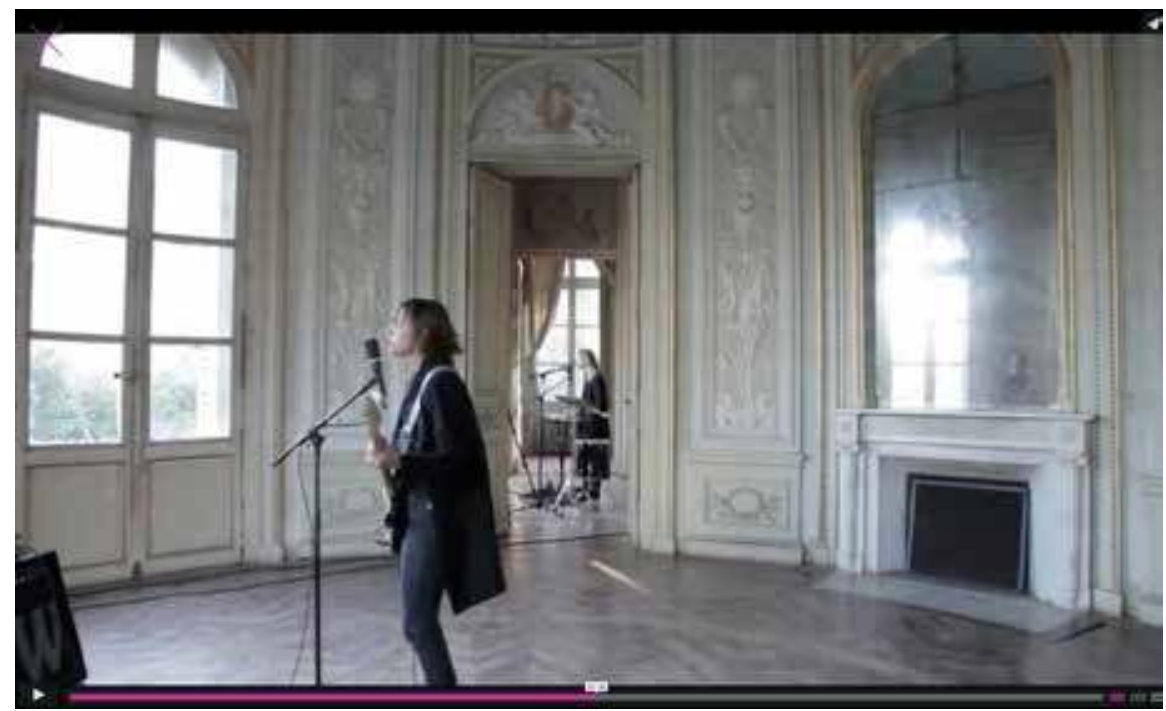

Figure 1 : Toulouse is beautiful:

IHR au Château de la Reynerie @ Mamy Scopitone \& Betty Book Production, 2014.

À l'inverse pour Lens ou Marseille, les réalisations misent sur une confrontation immédiate à l'image (fresque, photos des rues), et la bande-son consiste dès le début en bruits de ville: bourdonnement urbain, bruits de pas, de véhicules, bribes de conversations... Toutefois, alors qu'à Lens nul autre événement sonore n'intervient pour l'instant, à Marseille, dès la première station photographique, une voix se fait entendre: "Bienvenue ici. Ici, c'est-à-dire au cours Julien [...]. Je m'appelle Christophe, je suis l'auteur de cette promenade nocturne [...], à la recherche d'art éphémère [...] et d'histoires invraisemblables. Allons-y. Suivez-moi ». La bande-son procède tout de suite de la parole, celle de l'auteur de la promenade ${ }^{8}$, qui revêt le statut de passeur. Pourtant, cette construction sonore ne s'apparente pas à l'audio-guide, l'accompagnement oral n'y est pas déclamé, mais murmuré, intime: "Laissez traîner vos yeux sur les murs, je m'occupe de vos oreilles...».

Dans Toulouse is beautiful, au-delà du préalable de navigation web, le temps qui vient contraindre l'internaute est bien le seul temps du film, et le temps de consultation multimédia s'accorde avec celui de la durée de visionnage vidéo ${ }^{9}$ : il est régi par la bande-son, et est totalement homogène à la durée musicale. Le temps de consultation dans Lens, vous voyez le tableau est d'une tout autre nature, lent défilement de la fresque dessinée qui durera

8 Christophe Perruchi, musicien, compositeur, auteur de Promenade nocturne à Marseille et de la «promenade sonore» (Radio Grenouille), qui l'aura inspirée, http://www.promenades-sonores.com/audioguide/promenade-sonore/ le-souffleur.

9 Hors pause ou repositionnement avant ou arrière. 
26 minutes. Pauses et repositionnements avant ou arrière sont possibles, au gré de sous séquences, repérées en bas d'écran par des segments colorés en guise de barre de défilement (figure 2).

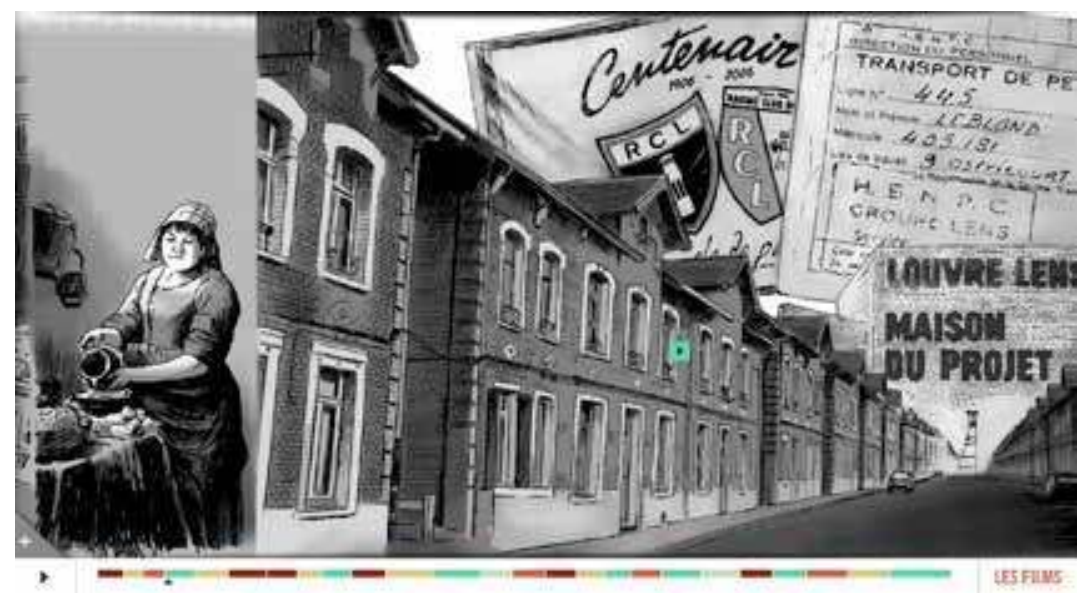

Figure 2 : Lens, vous voyez le tableau (c) Arte, Zorn Production International, musée du Louvre, 2012

Enfin, dans Promenade nocturne à Marseille, après la courte introduction vidéo, l'image se fige, et hors les interventions orales de notre guide ou le son d'ambiance diffusé en boucle, le temps de la consultation-visuelle- est suspendu. L'avancée dans l'univers urbain nécessitera une action de l'utilisateur. Les temporalités dans chacune de ces réalisations sont dissemblables, et introduisent différents statuts assignés à l'utilisateur. Car c'est bien une telle modalité, liée à l'interactivité, qui témoignera du déroulement hinc et nunc de ces productions (Julia, Smyrnaios, 2007). Ainsi, au-delà des image, son et temps, faudra-t-il que la consultation multimédia soit effectivement agie par l'utilisateur: il clique et s'engage dans un cheminement, qui s'avèrera-plus ou moins - interactif. Sur le site toulousain, une fois cliqué le bouton play, l'internaute-spectateur n'a plus besoin de contribuer: la vidéo défile, comme dans un média audiovisuel, et jusqu'à son terme, aucune intervention n'est requise. Dans Lens, vous voyez le tableau, au-delà d'une pause ou d'un repositionnement, quelques actions sont encore sollicitées de l'internaute-qui gagnera là le statut d'utilisateur. En cliquant sur l'un des boutons play insérés dans La fresque, il lance différentes séquences sonores (témoignages, conversations, bruits). Que l'utilisateur écoute aucune, plusieurs ou la totalité de ces digressions sonores ne change rien à la durée de consultation; et il pourra ne cliquer sur rien, il se cantonnera alors à la séquence des dessins. À Marseille, on l'a vu, la contribution de l'internaute s'avère nécessaire, sans quoi-plus comme dans un jeu vidéo que dans une vidéo - l'environnement photographique se fige, égrainant toutefois en boucle sa bande-son urbaine. L'utilisateur est plongé dans un street view nocturne, et son avancée est tributaire des clics exécutés, 
le pointeur de la souris se transformant en flèche directionnelle sur la chaussée: un chemin principal est proposé, balisé par une ligne verte guidant à travers le quartier (figure 3). Cependant, d'autres options sont possibles (dans les limites du périmètre investi par le dispositif). L'internaute pourra encore faire glisser l'image et modifier la vue à la façon d'un panoramique, pour regarder autour de lui et tourner à $360^{\circ}$ (gauche à droite et bas en haut): tout est donné à voir des rues de Marseille, charge à l'internaute d'agir, de prospecter.

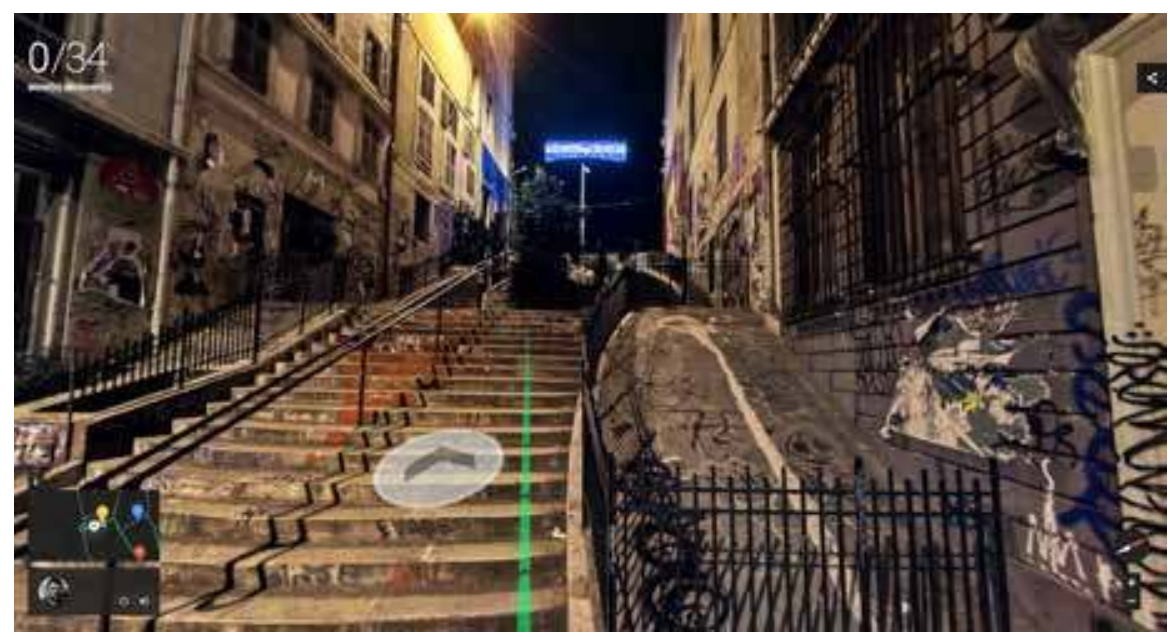

Figure 3 : Promenade nocturne à Marseille @ Google, 2014

Au cours de telles pérégrinations - qui deviennent alors celles d'un "spect-acteur» (Weissberg, 1999) - sont présentés, rapportés dans l'image de la rue, un certain nombre de boutons ou d'icônes cliquables, pour autant de «secrets tout au long du parcours». Ces «signes passeurs» (Souchier et al., 2003) amènent vers autant de discours de médiation, digressions ou contenus augmentés, aussi bien textuels, graphiques ou photographiques, vidéo, qu'oraux ou musicaux: ici une note ${ }^{10}$, évoquant Massalia, Marcel Pagnol, ou le savon de Marseille...; là un pochoir, ailleurs une improvisation jazz, plus loin la vidéo d'un graff en train de se faire... «Dans cette promenade en web immersif, on est en marche, et on se balade, on peut explorer - y'a une carte - y'a un côté un peu chasse au trésor [...] un peu safari urbain ${ }^{11}$ ». Dans le contexte d'une gamification (ludification) relative aux secteurs du multimédia, et plus largement à l'espace social, on pourra dire avec Sarah Labelle et Aude Seurrat que cette tendance cherche à «décrire l'adoption de mécanismes ludiques inscrits au sein de

10 Et ses renvois vers le moteur de recherche Google ou l'encyclopédie Wikipédia.

11 Marsactu, interview Julie de Muer, 14 avr. 2014, http://www.marsactu.fr/ culture/la-promenade-nocturne-une-experience-de-voyage-de-lordinaire-34300.html (propos à 4 min 21 s). 
situations et de pratiques sociales - ne relevant pas du jeu - avec pour objectif de favoriser l'engagement » (Labelle, Seurrat, 2014, 194).

Au final, deux façons pour l'internaute d'appréhender en ligne un patrimoine urbain se sont affirmées. D'une part, selon un mode plus passif, quand les contenus se dévoilent, s'affichent à ses yeux ou se manifestent spontanément à ses oreilles, et quand il n'aura plus qu'à se laisser porter par l'image de la caméra, se laisser emporter par la musique. D'autre part-mais la richesse du multimédia est aussi de pouvoir mêler les deux approches-, quand l'internaute pourra agir, explorer et découvrir à sa manière l'univers qui se présente, non plus campé dans une simple réception médiatique, mais dès lors s'employant à des interactions multimédias.

\section{2}

\section{Paysages artistiques : exprimer l'urbain autrement}

Chacun des dispositifs étudiés repose sur un regard aiguisé : la ville n'est pas présentée par le simple truchement d'une voix off qui commente l'historique et l'esthétique des monuments, mais elle est donnée à voir à travers le point de vue d'artistes. À Lens, Will Argunas croque le quotidien des habitants en noir et blanc et avec une précision naturaliste quasi-photographique, des scènes de genre composant une mosaïque sociale. Bandes dessinées et dessins à l'encre devenant ainsi l'élément central de ce carnet de ville, qui reprend la forme du "carnet de voyage" d'artiste, composé par le voyageur en itinérance. La visualisation des croquis fait parfois écho à la scénographie d'une exposition, la séquence «Nature morte au travail» illustre ainsi des visiteurs assistant-passivement? - derrière les cordons, à l'exposition des logos des grandes agences d'emploi ou d'intérim. Le regard que porte l'artiste est tout autant empreint d'esthétique que de réalisme sur le monde. À Toulouse, la caméra invite à observer les volutes méconnues de la basilique Saint-Sernin ou à admirer les rouages des machines de l'espace EDF-Bazacle. L'image animée esthétise le patrimoine et sert la partition musicale, par de lents mouvements et des alternances de plans intérieur-extérieur. À Marseille, l'image peu sophistiquée convient parfaitement au street art et au graff du quartier. Le propos photographique est ainsi de rendre concret et vivant un tel art urbain, comme en témoigne la séquence en accéléré montrant un artiste en train de graffer.

L'image, tout comme le son, donnent à voir le lieu selon un nouvel angle de vue au bénéfice du lieu patrimonial. Parmi les trois projets, la proposition la plus simple est celle de Toulouse is beautiful. La perspective esthétique se traduit dans le montage rythmé entre jeux acoustiques et regard cinématographique, pour mieux donner à voir des détails et des bâtiments ou monuments méconnus, qu'il s'agisse de l'hôtel d'Assézat (architecture historique), de la salle 
des machines de l'espace EDF-Bazacle (industrielle) ou des couloirs de l'hôpital La Grave (civile). Ce projet vise tout autant à montrer des lieux de la ville rose, que de donner à entendre les artistes sélectionnés (rythmes et mélodies composés par des groupes locaux), réalisation et production s'entremêlent, tout comme la médiation et la communication, dans la mesure où ce produit doit servir les musiciens dans une optique de promotion. Néanmoins, au-delà de cette commande, chaque artiste propose sa pâte, son style: Frédérika dans l'église Saint-Pierre des Chartreux s'inquiète des ambiances sonores et demande des prises de son différentes. À l'extérieur, le son est pris à la perche, puis peu à peu il monte à l'intérieur de l'église où la chanteuse joue sur la réverbération en chantant a cappella ${ }^{12}$. Et comme le rappelle Yves Jeanneret, "les lieux comptent singulièrement pour la musique, parce que l'atmosphère des volumes et la réverbération des surfaces la font exister, comme l'âme du violon et la table d'harmonie du piano" (Jeanneret, 2011, 59). Les lieux servent ainsi d'écrins à la production du son, contribuant concrètement à la médiation de la musique. Les internautes peuvent alors (re)découvrir la musique au gré d'images les invitant à écouter autrement.

Autre ambiance sonore, à Lens les séquences mêlent bruits, ambiances ou conversations issus du quotidien de la ville, et surtout les paroles des habitants; le regard est tourné vers la société et l'histoire. Quant à Marseille, les bruits et sons de la ville sont aussi à l'honneur: le projet trouve son origine dans des visites sonores de Julie de Muer proposées par Radio Grenouille, non pas au promeneur virtuel, mais à l'arpenteur bien réel de la cité phocéenne ${ }^{13}$. Christophe Perruchi, musicien-compositeur, est à l'origine de la création sonore, construite et composée à la façon d'une bande-son cinématographique ${ }^{14}$. Néanmoins, le projet est une véritable visite guidée, conçue comme une «mise en rapport des visiteurs avec une œuvre»(Gellereau, 2005, 9), fût-elle numérique, à la découverte de ce qu'on peut qualifier de «paysage sonore» selon Murray Schafer (1977). D'un point de vue cinématographique, le terme de landmark (marqueur sonore) renvoie au marquage territorial, à l'ancrage spatial du son, c'est un "son-territoire». L'internaute devient ainsi un "promeneur écoutant» (Chion, 1993) qui chemine dans le paysage, et découvre l'environnement acoustique de chaque cité: bruits de pas ou de véhicules, chants d'oiseaux, musique qui s'échappe d'une fenêtre, forment une composition urbaine.

Les arts sont à I'honneur dans les différents projets considérés. La ville est appréhendée sous ce prisme: que ce soit par La Liberté guidant le peuple qui donne le ton à la fresque de Lens puisque le tableau trône dans le parcours d'exposition, ou les graffs du cours Julien à Marseille, ou encore le hip hop du KKC-Orchestra à l'espace EDF-Bazacle de Toulouse. Ce sont donc tour à 
tour photographie, concert de jazz, graff, pop, dessin à l'encre de chine... qui dressent les contours de villes en recomposition ou en recréation. Les acteurs des différents projets, comme "les acteurs du musée» du Louvre décrits par Yves Jeanneret, «ne cessent $d$ 'inventer des manières de faire découvrir autrement les richesses du musée. Ils veulent faire en sorte que la confrontation à la singularité des œuvres ne signifie pas sacralisation de l'institution " (Jeanneret, 2011, 128). Le dispositif choisi, la mixité des formats et le croisement des arts sont autant d'éléments permettant de reconsidérer l'élargissement des publics ciblés, ainsi que leur posture de réception; ils servent de relais, de "passeurs» plus accessibles peut-être face à un patrimoine «légitime», pour une immersion culturelle. Dès lors, les concerts «en live», les dessins à l'encre comme les graffs prennent aussi une autre dimension, légitimés par le dispositif.

Ces productions mettent en œuvre une médiation culturelle à la fois de et par l'art. Expressions artistiques (musicales et graphiques) et médiation patrimoniale se complètent, dans une co-construction de sens. Le croisement des arts permet notamment de «stimuler des interprétations » et par là «donne la possibilité au visiteur de se construire sa propre appropriation " (Chaumier, 2010 , 35). Ces médiations artistiques peuvent ainsi être qualifiées de «synesthésiques et syncrétiques» (Lambert, Deramond, 2015), dans le sens où des formes d'art multisensorielles infusent les unes vers les autres, c'est-à-dire s'influencent mutuellement. C'est là l'une des clefs de voûte de cette médiation : jouer sur les sensations pour éprouver une expérience esthétique et créer des relations, des correspondances de(s) sens. De tels projets pourraient-ils infuser encore davantage pour s'apparenter aux transmédias, ces produits hybrides intégrant de manière "transmédiatique» (Jenkins, 2003) multimédia et supports ou formes d'expression diversifiés? La Galerie du temps à Lens, mêle déjà multimédia et bande dessinée, web-documentaire sur les travaux au Louvre-Lens, et web-série composée de petits films sur les œuvres exposées. Sans doute cette spécificité numérique est-elle une perspective pour concevoir la visite virtuelle, notamment de la ville: à la frontière entre les quartiers, les arts et les médias, pour multiplier les modalités de médiations à l'œuvre (sensible, artistique, ludique, documentaire) pour dire, montrer, faire entendre, la ville autrement.

\section{3 \\ Itinéraires de médiation: valoriser le patrimoine, révéler l'ordinaire}

Par le biais du support numérique et des expressions artistiques, Toulouse, Lens et Marseille sont "mises en scène» (Flon, 2012), voire «mises en communication» (Davallon, 2006, 37): le dispositif technique peut ainsi favoriser la découverte de l'insolite, de ce qui est habituellement caché ou passé sous silence, ou encore trop banal pour être remarqué (architectures peu connues, 
paroles d'habitants, façades de rues). De plus, le dispositif met en valeur des patrimoines urbains composites: certains extraordinaires de par leur valeur patrimoniale historique et d'autres, plus singulièrement, faisant écho à l'ordinaire et au quotidien urbain. Comment ce double mouvement de valorisation du patrimoine et de monstration de l'ordinaire se traduit-il? Et en écho aux mises en scène de la visite muséale ou patrimoniale (Gellereau, 2005), quelle relation au patrimoine ces visites virtuelles induisent-elles?

Les discours de médiation mobilisés permettent tout d'abord de mieux définir ce patrimoine. En effet, les médiations sont à la fois techniques et humaines; les médiateurs (et objets médiateurs) du patrimoine sont de trois types: des écrans de présentation tels des vues touristiques pour Toulouse, des témoignages d'habitants pour Lens et un réel médiateur, Christophe, qui prend en charge la visite guidée à Marseille. La valorisation du patrimoine passe ainsi par trois types de discours: un discours de communiquant, une parole d'habitant-témoin, et le récit d'un guide.

Dans le cas de Lens, cette valorisation du patrimoine passe notamment par les témoignages des habitants de la ville. Ils évoquent leur attachement à plusieurs types de patrimoines : urbain (monuments de la ville), paysage (terrils), industriel, humain, mais aussi leur rapport à la culture et à l'art. De plus, deux visions complémentaires de la notion de patrimoine coexistent ${ }^{15}$ : on observe la construction des deux séquences, l'une avec les témoignages des habitants (paroles de simple usager ou d'amateur d'art) dans La fresque, l'autre avec les témoignages des professionnels du musée (paroles d'experts) dans la séquence sur Les films présentant les enjeux de cette implantation du Louvre à Lens. Dans ce cas, ce sont essentiellement les "habitants-médiateurs» qui participent à la médiation en définissant ce qu'est selon eux le patrimoine. Ils acquièrent ainsi le "statut du guide témoin», construisant un "récit incarné » emprunt de légitimité de par sa «fonction authentificatrice»(Gellereau, 2000; 2005, 131). Dans les trois sites, une géographie sociale est donc redessinée par les témoignages des habitants (Lens) et des artistes (Toulouse, Marseille) sur leur cité.

De plus, le patrimoine mis en valeur se décline en trois facettes : il correspond d'une part à l'acception académique d'une valeur historique (monuments emblématiques, architectures, arts ou institutions classiques et légitimés) ; il s'apparente d'autre part à une vision plus contemporaine en mettant l'accent sur des créations artistiques parfois en marge (musique, dessin, graff) ; et il se décline enfin en une représentation d'un patrimoine plus immatériel ou vivant ${ }^{16}$

15 Voir l'analyse menée dans un quartier de Roubaix sur les visites par/pour les habitants d'une ville en reconversion: Gellereau M. (2011), Les multiples interprétations d'un patrimoine industriel: stratégies de valorisation et dispositifs de médiations, in Sciences de la Société, $n^{\circ} 78$, p. 97-112.

16 Selon la Convention pour la sauvegarde du patrimoine culturel immatériel (PCI) de l'UNESCO, 2003, le PCI traduit «les pratiques, représentations, expressions, connaissances, et savoir-faire- ainsi que les instruments, objets, artefacts et 
(ambiance sonore, vie de quartier, témoignages). Se mêlent ainsi des références patrimoniales reconnues (le Louvre, le Capitole et la basilique Saint-Sernin), avec d'autres plus insolites, inconnues, ou ordinaires (château de la Reynerie à Toulouse, patrimoine industriel à Lens, ou quartier marseillais et ses traces de street art). Représenter l'espace urbain (par le défilement de vues dessinées ou les déambulations), ou visualiser des lieux peu connus, voire inaccessibles pour le public (Toulouse), le processus de valorisation articule alors conjointement les idées de «rendre visible, produire de l'intelligible, mais également promouvoir» (Bonaccorci, Jarrigeon, 2014, 29). Cette valorisation du patrimoine sert donc aussi la visibilité d'un territoire, dans une visée communicationnelle.

Par ailleurs, nous pouvons voir ces sites web comme des supports de médiation et de médiatisation du patrimoine, participant d'un processus de mise en visibilité des objets et des situations, et amenant à interpréter et renouveler notre regard tout à la fois sur les villes concernées mais encore sur les arts mobilisés. À travers la mise en valeur d'un patrimoine «immatériel » (Bortolotto, 2011), c'est bien l'esprit des lieux qui est révélé. Comme le nom du site l'indique par exemple, la visite de Marseille est une "promenade nocturne», une balade à la rencontre de l'imprévu et des arts, un cheminement du flâneur qui, par cette expérience du corps, cette simulation d'une marche, amène aussi à une réflexion sur les lieux. C'est à ce regard de flâneur qu'invite l'esthétique des vidéos toulousaines: prendre le temps de s'approprier l'espace monumental, au rythme des sons, pour laisser courir l'imagination. Il s'agit donc bien d'itinéraires dans ces découvertes du patrimoine urbain: le parcours est d'une certaine façon balisé par un contenu qui, même interactif, ne laisse que peu de place à une libre déambulation. Pour Toulouse, les séquences proposées s'apparentent à un circuit touristique, pour Lens c'est le rythme du défilement de la fresque qui scandera le pas, et seul l'exemple de Marseille propose un dispositif capable de simuler cette idée de liberté de cheminement. Nous pouvons identifier dans le corpus trois représentations et expériences spatiales qui incarnent autant de modalités de médiation: un lieu visualisé (catalogue ou book audiovisuel toulousain traduisant une pause perceptive, l'immobilité), un paysage sonore (fresque lensoise suggérant métaphoriquement une marche, le déplacement), et un espace visité (expérience immersive marseillaise symbolisant un cheminement, la découverte).

Il s'agit ensuite de faire la médiation du commun, du quotidien, en mettant l'accent sur ce qui n'est certes pas le plus emblématique, mais qui contribue néanmoins à construire une identité urbaine, voire culturelle, comme Anne Watremez a pu l'analyser en montrant en quoi une pratique ordinaire de la ville par ses habitants peut modifier ou inventer un patrimoine (Watremez, 2008,

espaces culturels qui leur sont associés-que les communautés, les groupes et, le cas échéant, les individus reconnaissent comme faisant partie de leur patrimoine culturel[...]», http://www.unesco.org/culture/ich/index.php?l$g=f r \& p g=00003$. 
12). Dans cet esprit, Julie de Muer relate le projet de Marseille: «Dans les promenades sonores, on a une impression de dépaysement y compris lorsque l'on connaît ces quartiers. Ce sont des voyages tout à côté, basés sur la rencontre avec l'autre, qu'il soit l'habitant ou l'artiste ${ }^{17}$ ». Ces villes en live nous proposent ainsi d'explorer des univers à la fois proches et lointains, connus et inconnus, des objets patrimoniaux ou d'autres plus triviaux et quotidiens, et d'une certaine manière invitent à être attentifs aux détails. La médiation porte alors sur des objets et monuments ordinaires ou quotidiens, elle met en valeur un «patrimoine de proximité18 » (Chunikhina, 2013) accessible à tous. Le thème de l'ordinaire et du familier ou, concernant le patrimoine urbain son caractère d'«invisible ordinaire», mis en lumière par Paul Veyne (1988), s'exprime ici principalement à travers la bande-son (Lens et Marseille) et souvent par des détails-archétypes de l'urbain - (bruits d'ambiance, bruits de rue, voix) qui révèlent une réalité somme toute assez banale que Georges Perec nomme "l'infra-ordinaire». Cette dimension sonore incarne une identité urbaine et une idée de vie: la ville est comme saisie sur le vif, vivante. Yves Jeanneret souligne que «les médias ne laissent pas intacte la topographie des lieux de culture» $(2011,105)$, et il évoque comment, dans notre société, les médias (numériques et interactifs en premier lieu) reconfigurent l'ici, le partout et le nulle part. Nous pourrions aussi préciser que les sites web analysés proposent encore un ailleurs, entre des éléments réels (lieux qui existent) et des recompositions imaginaires (espaces pratiqués et recréations artistiques). La possibilité offerte par ces itinéraires numériques est bien celle d'appréhender ensemble projet artistique et ville en situation, parcours concret et visée imaginaire ${ }^{19}$.

\section{4 Conclusion}

À travers ces trois sites web, nous avons analysé des parcours de médiation singuliers où les techniques (clip vidéo, bande son ou street view), élargissent les modalités de visite virtuelle, comme autant de manières de donner à voir et à entendre le patrimoine urbain, constituant par là même de véritables paysages, visuels autant que sonores (des paysages que l'on écoute ou des sons qui se dessinent). Ces paysages sont l'expression d'une identité urbaine et d'une forme de "patrimonialité», entendue comme "le rapport d'attachement des pratiquants ordinaires d'une ville avec ce qu'ils considèrent comme leur patri-

17 Marsactu, op. cit

18 Autrement dénommé «petit patrimoine»; l'expression désigne, depuis les années 1990, des formes patrimoniales reconnues «d'intérêt local», mais non classées, non inscrites, et non protégées par l’État.

19 Comme le note J. de Muer, op.cit.: «On découvre ce quartier, mais on le "fictionne" aussi. Ce mélange de rêve et réalité colle bien avec la pratique du pochoir, cet espèce de surajout assez ténu sur la réalité qui nous entoure». 
moine» (Watremez, 2008, 12). Cette notion de patrimonialité peut se traduire tout à la fois par les paroles des habitants ou d'un guide (Lens et Marseille), ou encore le lien suscité avec les internautes.

Nous avons aussi montré comment l'hybridation des formes et des médias liés au numérique, associée au croisement des arts mobilisés, participent à construire des médiations renouvelées, et peuvent façonner un autre regard sur les patrimoines, qu'ils soient matériels et immatériels, issus des genres monumentaux, picturaux ou musicaux. L'expression artistique crée ainsi des chemins de traverse pour raconter, traverser ou revisiter des patrimoines, des mémoires et des territoires (Lambert, Trouche, 2011).

Ces itinéraires numériques nous ont enfin amenés à réfléchir sur l'art et la manière de valoriser le patrimoine urbain auprès du public en ligne, voire de reconsidérer cette notion, en décentrant le regard depuis un patrimoine historique ou sacralisé vers l'ordinaire et le quotidien, du passé vers le présent, et du singulier vers le commun, autrement dit d'un patrimoine qui se caractérise par son exception à celui que nous avons certes en commun, mais dans sa singulière banalité: les sites web valorisent des patrimoines anciens, mais aussi ce qui se crée et s'exprime, même de façon éphémère, au présent de la ville. Considérant, avec Jean Davallon, «que le passé n'existe que comme construit dans le présent» $(2006,27)$, nous pouvons aussi envisager que ces mises en communication représentent un patrimoine en devenir. À l'image de la photographie qui «constitue une médiation dans la construction d'un rapport au passé pour des territoires en devenir patrimonial» (Tardy, 2011, 94)-comme le sont les paysages - les supports numériques étudiés deviennent en eux-mêmes des documents ayant d'une part une valeur de médiation documentaire (Tardy, 2014), et d'autre part une valeur patrimoniale, dans la mesure où ils conservent, exposent et transmettent un «ça a été» barthésien, un espace et un temps qui symbolisent la ville en live. 
Bibliographie

Bonaccorci J., Jarrigeon A. (2014). "Visualisations urbaines et partage des représentations". In Communication \& Langages, $n^{\circ} 180$, p. 25-30.

Bortolotto C. (2011). Le patrimoine culturel immatériel, enjeux d'une nouvelle catégorie, Paris, Éditions de la Maison des sciences de l'homme.

ChaumierS. (2010). "La muséographie de l'art, ou la dialiectique de l'œuvre et de se réception ". In Culture \& Musées, $n^{\circ} 10$, p. 21-43.

Chion M. (1993). Le promeneur écoutant. Essais d'acoulogie, Paris, Plume.

Chunikhinal. (2013). "Le 'patrimoine de proximité»: du "coup de cœur' au label». In FabreD., (dir.), Émotions patrimoniales, Paris, Éditions de la Maison des sciences de l'homme, p.175-194.

Davallon J. (2006). Le don du patrimoine: une approche communicationnelle de la patrimonialisation, Paris, Hermès Sciences-Lavoisier.

FlonE. (2012). Les mises en scène du patrimoine: savoir, fiction et médiation, Paris, Hermès Lavoisier.

Gellereau M. (2000). "Le récit incarné de la visite guidée». In Caune J. et DufrêneB., (dir.), Médiations du corps, actes du colloque, 24-25nov.2000, Grenoble,
Université Stendhal, p.101-109.

Gellereau M. (2005). Les mises en scène de la visite guidée. Communication et médiation, Paris, L'Harmattan.

Jenkins H. (2003). «Transmedia Storytelling". In MIT Technology Review, http://www.technologyreview.com/ news/401760/transmedia-storytelling/, consulté le 4 mars 2015.

Jeanneret Y. (2011). Where is Monna Lisa? et autres lieux de culture, Paris, Le Cavalier Bleu.

Julia J.-Th., Smyrnaios N. (2007). "Figures d'un cheminement, ou 'Ceci n'est pas un parcours multimédia' ». In Les Cahiers de Champs Visuels, $n^{\circ} 3$, p.11-42.

LabelleS., Seurrat A. (2014). "Serious game et réécriture des savoirs: hétérogénéité des médiations documentaires». In Boustany J., BroudouxE. et Chartron Gh. (dir.), La médiation numérique, Bruxelles, De Boeck, p.193-205.

LambertE., Deramond J. (2015). Mosaïque des arts en médiations: synesthésie et syncrétisme au musée des Augustins, Journée d'étude $\mathrm{OMCl}$, Toulouse, musée des Augustins, mai 2014, Toulouse, Cépaduès (à paraître).

LambertE., TroucheD.(dir.), (2011, daté 2009). "Art, mémoire et territoire: du fragment à l'entremêlement, I'anamnèse comme processus". In Sciences de la Société, $n^{\circ} 78$, p.3-11. 
Murray Schafer R. (1977, rééd. 2010).

Le paysage sonore. Le monde comme musique, Marseille, Wildproject.

SouchierE., Jeanneret Y., Le Marec J.(dir.), (2003). Lire, écrire, récrire. Objets, signes et pratiques des médias informatisés, Paris, Bpi/Centre Pompidou.

Tardy C. (2011). «L'expérience visuelle du temps: photographie et territoire du patrimoine". In Sciences de la Société, n78, p.79-95.

Tardy C. (dir.), (2014). Les médiations documentaires des patrimoines, Paris, L'Harmattan.

VeyneP. (1988). "Conduites sans croyance et œuvres d'art sans spectateurs". In Diogène, n¹43, p.3-22.

WatremezA. (2008). «Vivre le patrimoine urbain au quotidien: pour une approche de la patrimonialité ». In Culture \& Musées, $\mathrm{n}^{\circ} 11, \mathrm{p} .11-36$.

Weissberg J.-L. (1999). Présences à distance, Paris, L'Harmattan. 